Article

\title{
Three New Classes of Solvable N-Body Problems of Goldfish Type with Many Arbitrary Coupling Constants
}

\author{
Francesco Calogero ${ }^{1,2}$
}

1 Physics Department, University of Rome "La Sapienza", 00185 Rome, Italy; francesco.calogero@roma1.infn.it 2 Istituto Nazionale di Fisica Nucleare, Sezione di Roma, 00185 Rome, Italy

Academic Editor: Charles F. Dunkl

Received: 31 March 2016; Accepted: 17 June 2016; Published: 24 June 2016

\begin{abstract}
Three new classes of $N$-body problems of goldfish type are identified, with $N$ an arbitrary positive integer $(N \geqslant 2)$. These models are characterized by nonlinear Newtonian ("accelerations equal forces") equations of motion describing $N$ equal point-particles moving in the complex $z$-plane. These highly nonlinear equations feature many arbitrary coupling constants, yet they can be solved by algebraic operations. Some of these $N$-body problems are isochronous, their generic solutions being all completely periodic with an overall period $T$ independent of the initial data (but quite a few of these solutions are actually periodic with smaller periods $T / p$ with $p$ a positive integer); other models are isochronous for an open region of initial data, while the motions for other initial data are not periodic, featuring instead scattering phenomena with some of the particles incoming from, or escaping to, infinity in the remote past or future.
\end{abstract}

Keywords: solvable many-body problems; integrable many-body problems; integrable dynamical systems; solvable many-body problems of goldfish type

\section{Introduction}

Recently, a new technique to identify many-body problems solvable by algebraic operations has been introduced [1,2], and several examples of such models have been discussed [1-6]. In the present paper, three new classes of such models are introduced and discussed (the impatient reader may immediately glance at these findings reported in the next section). We term these models "of goldfish type" because their Newtonian ("accelerations equal forces") equations of motion read as follows:

$$
\begin{aligned}
& \ddot{z}_{n}=(2 r+1) \mathbf{i} \omega \dot{z}_{n}+r(r+1) \omega^{2} z_{n} \\
& -\sum_{\ell=1, \ell \neq n}^{N}\left[\frac{\left(\dot{z}_{n}+\mathbf{i} r \omega z_{n}\right)\left(\dot{z}_{\ell}+\mathbf{i} r \omega z_{\ell}\right)}{z_{n}-z_{\ell}}\right]+f_{n}(\vec{z}, \dot{z}),
\end{aligned}
$$

with the nonlinear functions $f_{n}(\vec{z}, \dot{\vec{z}})$ appropriately defined (see below). (The original goldfish model is the special case of these equations of motion with $r=0$ and $f_{n}(\vec{z}, \dot{\vec{z}})=0$; after its first identification as a solvable model [7], and its tentative recognition as a "goldfish" [8], this $N$-body problem and some of its extensions have been investigated in several publications (see, for instance, [9-20]).

Notation 1.1. Above, and hereafter, $N$ is an arbitrary positive integer $(N \geqslant 2)$; indices such as $n, m, \ell$ run over the positive integers from 1 to $N$ (unless otherwise indicated: see, for instance, the restriction on the values of $\ell$ in (1)); the $N$ complex coordinates $z_{n} \equiv z_{n}(t)$ identify the positions of $N$ points moving 
in the complex z-plane as functions of the time $t$; below, we also introduce other complex variables $\zeta_{n} \equiv \zeta_{n}(\tau)$ that depend on the complex variable $\tau$ related to the real variable $t$ ("time") as follows:

$$
\tau \equiv \tau(t)=\frac{\exp (\mathbf{i} \omega t)-1}{\mathbf{i} \omega} .
$$

Above, and hereafter, $\mathbf{i}$ is the imaginary unit $\left(\mathbf{i}^{2}=-1\right), \omega$ is an arbitrary (nonvanishing) real constant, so that $\tau(t)$ vanishes at the initial time $t=0$ and is periodic in time with period $T_{0}$,

$$
\tau(0)=0, \quad \tau\left(t+T_{0}\right)=\tau(t), \quad T_{0}=\frac{2 \pi}{|\omega|} .
$$

Above, and hereafter, we adopt the standard notation according to which superimposed dots denote differentiations with respect to the time variable $t$, so that, for instance,

$$
\dot{\tau} \equiv \dot{\tau}(t)=\exp (\mathbf{i} \omega t)
$$

while primes appended to a function indicate differentiations with respect to the argument of that function (see, for instance, (3b) and (3c), where the relevant variable is of course $\tau$ ). The auxiliary complex coordinates $\zeta_{n} \equiv \zeta_{n}(\tau)$ are related to the $N$ complex coordinates $z_{n} \equiv z_{n}(t)$-which are the main protagonists of our $N$-body problems, as they identify the positions of $N$ equal unit-mass point-particles moving in the complex z-plane according to the Newtonian ("accelerations equal forces") equations of motion of type (1) - by the following relations:

$$
z_{n}(t)=\exp (\mathbf{i} r \omega t) \zeta_{n}(\tau), \zeta_{n}(\tau)=\exp (-\mathbf{i} r \omega t) z_{n}(t),
$$

implying

$$
\begin{gathered}
\dot{z}_{n}=\mathbf{i} r \omega z_{n}+\exp [\mathbf{i}(r+1) \omega t] \zeta_{n}^{\prime} \\
\zeta_{n}^{\prime}=\exp [-\mathbf{i}(r+1) \omega t]\left(\dot{z}_{n}-\mathbf{i} r \omega z_{n}\right), \\
\ddot{z}_{n}=\mathbf{i}(2 r+1) \omega \dot{z}_{n}+r(r+1) \omega^{2} z_{n}+\exp [\mathbf{i}(r+2) \omega t] \zeta_{n}^{\prime \prime} \\
\zeta_{n}^{\prime \prime}=\exp [-\mathbf{i}(r+2) \omega t]\left[\ddot{z}_{n}-\mathbf{i}(2 r+1) \omega \dot{z}_{n}-r(r+1) \omega^{2} z_{n}\right] .
\end{gathered}
$$

Above, and hereafter, the number $r$ is required to be real and rational,

$$
r=\frac{q}{p}
$$

where, of course, the numerator $q$ and the denominator $p$ are coprime integers, and, for definiteness, we hereafter assume that $p$ is positive, $p \geqslant 1$.

Finally, note that above we often omitted indicating explicitly the argument of functions, and we will do so throughout whenever this can be done without causing confusion.

In this paper, a key role is played by the following monic $\tau$-dependent polynomial of degree $N$ in the (complex) variable $\zeta$,

$$
p_{N}(\zeta ; \underline{\zeta}(\tau) ; \vec{\gamma}(\tau))=\prod_{n=1}^{N}\left[\zeta-\zeta_{n}(\tau)\right]=\zeta^{N}+\sum_{m=1}^{N}\left[\gamma_{m}(\tau) \zeta^{N-m}\right]
$$


which features the $N$ zeros $\zeta_{n}(\tau)$ and the $N$ coefficients $\gamma_{m}(\tau)$; and by its counterpart,

$$
P_{N}(z ; \underline{z}(t) ; \vec{w}(t))=\prod_{n=1}^{N}\left[z-z_{n}(t)\right]=z^{N}+\sum_{m=1}^{N}\left[w_{m}(t) z^{N-m}\right]
$$

featuring the $N$ zeros $z_{n}(t)$ and the $N$ coefficients $w_{m}(t)$ (see Notation 1.1).

Notation 1.2. The $N$ coefficients $\gamma_{m} \equiv \gamma_{m}(\tau)$ of the polynomial (4a) are of course expressed in terms of its $N$ zeros $\zeta_{n} \equiv \zeta_{n}(\tau)$ via the formulas

$$
\begin{gathered}
\gamma_{m}=(-1)^{m} \sigma_{m}(\underline{\zeta}), \\
\sigma_{m}(\underline{\zeta})=\frac{1}{m !} \sum_{n_{1}, n_{2}, \ldots, n_{m}=1}^{N} *\left(\zeta_{n_{1}} \zeta_{n_{2}} \cdots \zeta_{n_{m}}\right) .
\end{gathered}
$$

Above, and hereafter, the symbol $\underline{\zeta}$ denotes the unordered set of the $N$ complex numbers $\zeta_{n}$ (and likewise $\underline{z}$ is the unordered set of the $N$ complex numbers $z_{n}$ ), while $\vec{\gamma}$ (see (4a) and below) is the $N$-vector with components $\gamma_{m}$ (and likewise $\vec{w}$ is the $N$-vector with components $w_{m}$ ). The symbol $\sum_{n_{1}, n_{2}, \ldots, n_{m}}^{N} *$ denotes the sum from 1 to $N$ over the $m$ indices $n_{1}, n_{2}, \ldots, n_{m}$ with the restriction that these indices be all different among themselves, so that for $N=2$,

$$
\gamma_{1}=-\left(\zeta_{1}+\zeta_{2}\right), \gamma_{2}=\zeta_{1} \zeta_{2}
$$

for $N=3$,

$$
\gamma_{1}=-\left(\zeta_{1}+\zeta_{2}+\zeta_{2}\right), \gamma_{2}=\zeta_{1} \zeta_{2}+\zeta_{2} \zeta_{3}+\zeta_{3} \zeta_{1}, \gamma_{3}=-\zeta_{1} \zeta_{2} \zeta_{3}
$$

and so on. Let us also display the corresponding formulas for the $\tau$-derivative of $\gamma_{m} \equiv \gamma_{m}(\tau)$,

$$
\begin{aligned}
& \gamma_{m}^{\prime}=(-1)^{m} \sum_{n=1}^{N}\left[\zeta_{n}^{\prime} \sigma_{n, m}\left(\zeta_{n} ; \underline{\zeta}\right)\right], \\
& \sigma_{n, 1}\left(\zeta_{n} ; \underline{\zeta}\right)=1, \\
& \sigma_{n, m}\left(\zeta_{n} ; \underline{\zeta}\right)=\frac{1}{(m-1) !} \sum_{n_{1}, n_{2}, \ldots, n_{m-1}=1}^{N} * n *\left(\zeta_{n_{1}} \zeta_{n_{2}} \cdots \zeta_{n_{m-1}}\right), \\
& m=2, \ldots, N,
\end{aligned}
$$

where the symbol $\sum_{n_{1}, n_{2}, \ldots, n_{m}}^{N} * n *$ denotes the sum from 1 to $N$ over the $m$ indices $n_{1}, n_{2}, \ldots, n_{m}$ with the restriction that these indices be all different among themselves and all different from the index $n$, so that for $N=2$,

$$
\gamma_{1}^{\prime}=-\left(\zeta_{1}^{\prime}+\zeta_{2}^{\prime}\right), \gamma_{2}^{\prime}=\zeta_{1}^{\prime} \zeta_{2}+\zeta_{1} \zeta_{2}^{\prime}
$$

for $N=3$,

$$
\begin{aligned}
& \gamma_{1}^{\prime}=-\left(\zeta_{1}^{\prime}+\zeta_{2}^{\prime}+\zeta_{2}^{\prime}\right), \\
& \gamma_{2}^{\prime}=\zeta_{1}^{\prime}\left(\zeta_{2}+\zeta_{3}\right)+\zeta_{2}^{\prime}\left(\zeta_{3}+\zeta_{1}\right)+\zeta_{3}^{\prime}\left(\zeta_{1}+\zeta_{2}\right), \\
& \gamma_{3}^{\prime}=-\left(\zeta_{1}^{\prime} \zeta_{2} \zeta_{3}+\zeta_{2}^{\prime} \zeta_{3} \zeta_{1}+\zeta_{3}^{\prime} \zeta_{1} \zeta_{2}\right),
\end{aligned}
$$

and so on. Analogous formulas hold, of course, for the relations among the $N$ coefficients $w_{m} \equiv w_{m}(t)$ and the $N$ zeros $z_{n} \equiv z_{n}(t)$ of the polynomial (4b), and their time-derivatives; for instance, 


$$
w_{m}=\frac{(-1)^{m}}{m !} \sum_{n_{1}, n_{2}, \ldots, n_{m}=1}^{N} *\left(z_{n_{1}} z_{n_{2}} \cdots z_{n_{m}}\right) .
$$

Let us also report the relations-implied by (3a)—among the $N$ coefficients $w_{m}(t)$ of the polynomial (4b) and the $N$ coefficients $\gamma_{m}(\tau)$ of the polynomial (4a)

$$
w_{m}(t)=\exp (\mathbf{i} m r \omega t) \gamma_{m}(\tau),
$$

with $\tau$ related to $t$ via (2) and $r$ defined by (3d), implying

$$
\begin{aligned}
& \dot{w}_{m}(t)=\mathbf{i} m r \omega w_{m}(t)+\exp [\mathbf{i}(m r+1) \omega t] \gamma_{m}^{\prime}(\tau), \\
& \gamma_{m}^{\prime}(\tau)=\exp [-\mathbf{i}(m r+1) \omega t]\left[\dot{w}_{m}(t)-\mathbf{i} m r \omega w_{m}(t)\right] . \\
& \ddot{w}_{m}(t)=\mathbf{i}(2 m r+1) \omega \dot{w}_{m}(t)+m r(m r+1) \omega^{2} w_{m}(t) \\
& +\exp [\mathbf{i}(m r+2) \omega t] \gamma_{m}^{\prime \prime}(\tau) \\
& \gamma_{m}^{\prime \prime}(\tau)=\exp [-\mathbf{i}(m r+2) \omega t]\left[\ddot{w}_{m}(t)\right. \\
& \left.-\mathbf{i}(2 m r+1) \omega \dot{w}_{m}(t)-m r(m r+1) \omega^{2} w_{m}(t)\right] .
\end{aligned}
$$

Remark 1.1. Two comments on the problem to determine the $N$ zeros $\zeta_{n}$, respectively, $z_{n}$ of a monic polynomial of degree $N$ in $\zeta$, respectively, $z$ from its $N$ coefficients $\gamma_{m}$, respectively, $w_{m}$ (see (4a), respectively, (4b)).

(i) Of course, the assignment of the $N$ coefficients of a polynomial defines uniquely the corresponding unordered set of its $N$ zeros, but generally it only allows to compute explicitly these $N$ zeros for $N \leqslant 4$.

(ii) Moreover-and quite relevantly in our context (see below) - if a polynomial features a dependence on an additional variable (as, for instance, the dependence of the polynomial $P_{N}(z ; \underline{z}(t) ; \vec{w}(t))$ on the real variable $t$ ("time") (see (4b)), then the unordered character of the set of its $N$ zeros $z_{n}(t)$ is generally only relevant at one value of time, say at the "initial" time $t=0$, since, at other values of time, the ordering gets generally determined by the natural requirement that the functions $z_{n}(t)$ evolve continuously over time. This prescription then fixes, for all time, the ordering of the zeros $z_{n}(t)$-i.e., the assignment of the value $n$ of its index to each zero $z_{n}(t)$ 一as long as the coefficients $w_{m}(t)$ evolve themselves continuously and unambiguously over time and moreover no "collision" of two or more zeros occurs over the time evolution, i.e., for all time $z_{n}(t) \neq z_{\ell}(t)$ if $n \neq \ell$ (since clearly such collisions imply a loss of identity of the coinciding zeros). However, this identification requires an analysis of the time evolution of the $N$ zeros $z_{n}(t)$ not only in the complex $z$-plane, but in fact over the $N$-sheeted Riemann surface associated to the $N$ roots of the polynomial $P_{N}(z ; \underline{z}(t) ; \vec{w}(t))$, and/or over the evolution of each coefficient $w_{m}(t)$ if its time evolution takes itself place on a Riemann surface (as it indeed happens in the cases discussed below).

A key formula for the identification and investigation of solvable Newtonian $\mathrm{N}$-body problems is the following relation [1] among the $\tau$-evolutions of the $N$ zeros and the $N$ coefficients of the monic polynomial $p_{N}(\zeta ; \tau)$ (see $\left.4 \mathrm{a}\right)$ ) of degree $N$ in its argument $\zeta$ and depending on the extra variable $\tau$ :

$$
\zeta_{n}^{\prime \prime}=\sum_{\ell=1 ; \ell \neq n}^{N}\left(\frac{2 \zeta_{n}^{\prime} \zeta_{\ell}^{\prime}}{\zeta_{n}-\zeta_{\ell}}\right)-\left[\prod_{\ell=1 ; \ell \neq n}^{N}\left(\zeta_{n}-\zeta_{\ell}\right)^{-1}\right] \sum_{m=1}^{N}\left[\gamma_{m}^{\prime \prime}\left(\zeta_{n}\right)^{N-m}\right] .
$$


In the present paper-which is a sequel to [6], so that we dispense below from some of the remarks reported there about the significance of the solvable many-body models treated herein-we focus on the $N$-body models that obtain via (9) for the coordinates $\zeta_{n}(\tau)$-and especially for the corresponding coordinates $z_{n}(t)$ (see (3)) —when the coefficients $\gamma_{m}(\tau)$ evolve according to the following system of decoupled nonlinear Ordinary Differential Equations (ODEs):

$$
\gamma_{m}^{\prime \prime}=g_{m}\left(\gamma_{m}^{\prime}\right)^{a_{m}}\left(\gamma_{m}\right)^{b_{m}}
$$

where the $N$ "coupling constants" $g_{m}$ are arbitrary (possibly complex), while the two (sets of) parameters $a_{m}$ and $b_{m}$ are instead hereafter required to be real rational numbers and to be related to each other (and to the parameter $r$, (see (3d)) by the single relation:

$$
a_{m}=2-\frac{\left(1+b_{m}\right) m r}{1+m r}, b_{m}=1-a_{m}+\frac{2-a_{m}}{m r},
$$

which-provided neither $r$ nor $1+m r$ vanish, i.e., $r \neq 0$ and $r \neq-1 / m$ for $m$ in its range from 1 to $N$, as we now assume (the case $r=0$ shall be discussed below separately) -is necessary and sufficient to guarantee that the corresponding system of ODEs satisfied by the coefficients $w_{m}(t)$ be autonomous, reading as follows (as the diligent reader will easily verify via (10a) and (8)) :

$$
\begin{aligned}
\ddot{w}_{m}(t)= & \mathbf{i}(2 m r+1) \omega \dot{w}_{m}(t)+m r(m r+1) \omega^{2} w_{m}(t) \\
& +g_{m}\left(\dot{w}_{m}-\mathbf{i} m r \omega w_{m}\right)^{a_{m}}\left(w_{m}\right)^{b_{m}}
\end{aligned}
$$

In fact, we will focus below only on certain specific assignments of the parameters $r, a_{m}, b_{m}$ which allow the explicit solution of the ODEs (10a) in terms of elementary functions. The corresponding solvable $N$-body models satisfied by the coordinates $z_{n} \equiv z_{n}(t)$ are displayed-and their properties discussed-in the following Section 2, with the corresponding proofs provided in Section 3, while the special case with $a_{m}=2$ and $b_{m}=-1$ (and with $g_{m}$ an arbitrary rational number) is treated in [6].

\section{Results}

The Newtonian equations of motion of the first class of $\mathrm{N}$-body models treated in this paper read as follows:

$$
\begin{aligned}
& \ddot{z}_{n}=\mathbf{i}(2 r+1) \omega \dot{z}_{n}+r(r+1) \omega^{2} z_{n} \\
& +\sum_{\ell=1, \ell \neq n}^{N}\left[\frac{2\left(\dot{z}_{n}-\mathbf{i} r \omega z_{n}\right)\left(\dot{z}_{\ell}-\mathbf{i} r \omega z_{\ell}\right)}{z_{n}-z_{\ell}}\right]-\left[\prod_{\ell=1, \ell \neq n}^{N}\left(z_{n}-z_{\ell}\right)^{-1}\right] \\
& \cdot \sum_{m=1}^{N}\left[g_{m}\left(\dot{w}_{m}-\mathbf{i} m r w_{m}\right)^{1+[1 /(1+m r)]}\left(z_{n}\right)^{N-m}\right]
\end{aligned}
$$

with $r$ an arbitrary rational number (see (3d); of course $r \neq-1 / m$ for $m=1,2, \ldots, N$ if $g_{m} \neq 0$ ), $\omega$ an arbitrary nonvanishing real number, the $N$ coupling constants $g_{m}$ arbitrary complex numbers (not all vanishing) and (see Notation 1.2)

$$
\begin{gathered}
w_{m}=\frac{(-1)^{m}}{m !} \sum_{n_{1}, n_{2}, \ldots, n_{m}=1}^{N} *\left(z_{n_{1}} z_{n_{2}} \cdots z_{n_{m}}\right), \\
\dot{w}_{m}=\frac{(-1)^{m}}{(m-1) !} \sum_{n=1}^{N}\left\{\dot{z}_{n}\left[\sum_{n_{1}, n_{2}, \ldots, n_{m-1}=1}^{N} * n *\left(z_{n_{1}} z_{n_{2}} \cdots z_{n_{m}}\right)\right]\right\} .
\end{gathered}
$$


We then assert that this $\mathrm{N}$-body model is solvable by algebraic operations, its remarkable properties being detailed by the following

Proposition 2.1. The $N$ complex coordinates $z_{n}(t)$ providing the solution at time $t$ of the initial-values problem of the Newtonian equations of motion (11) are the $N$ zeros of the monic polynomial (4b), the $N$ coefficients $w_{m}(t)$ of which are given, in terms of the initial data $z_{n}(0), \dot{z}_{n}(0)$, by the following formulas:

$$
\begin{gathered}
w_{m}(t)=\exp (\mathbf{i} r m \omega t) \gamma_{m}(\tau(t)), \tau(t)=\frac{\exp (\mathbf{i} \omega t)-1}{\mathbf{i} \omega}, \\
\gamma_{m}(\tau)=\left[w_{m}(0)+B_{m}^{(1)}\right]\left(1-\frac{\tau}{\check{\tau}_{m}^{(1)}}\right)^{-m r}-B_{m}^{(1)}, \\
\check{\tau}_{m}^{(1)}=\frac{m r\left\{A_{m}\left[w_{m}(0)+B_{m}^{(1)}\right]\right\}^{-1 /(m r)}}{A_{m}}, \\
B_{m}^{(1)}=\frac{\left[\dot{w}(0)-\mathbf{i} m r \omega w_{m}(0)\right]^{(m r) /(1+m r)}}{A_{m}}-w_{m}(0), \\
w_{m}(0)=\frac{(-1)^{m}}{m !} \sum_{n_{1}, n_{2}, \ldots, n_{m}=1}^{N} *\left[z_{n_{1}}(0) z_{n_{2}}(0) \cdots z_{n_{m}}(0)\right] \\
\dot{w}_{m}(0)=\frac{(-1)^{m}}{(m-1) !} \sum_{n=1}^{N}\left\{\dot{z}_{n}(0) \sum_{n_{1}, n_{2}, \ldots, n_{m-1}=1}^{N} * n *\left[z_{n_{1}}(0) z_{n_{2}}(0) \cdots z_{n_{m-1}}(0)\right]\right\} .
\end{gathered}
$$

These functions $w_{m}(t)$ are, of course, defined by continuity in $t$ from their initial values $w_{m}(0)$ (i.e., this prescription identifies the determination of all the rational roots appearing in the above formulas), and note that, if $g_{m}=0$, the formula (12b) must be replaced by

$$
\gamma_{m}(\tau)=w_{m}(0)+\left[\dot{w}(0)-\mathbf{i} m r \omega w_{m}(0)\right] \tau .
$$

Let us also report the properties of these solutions, (12), when they are generated by generic initial data, $z_{n}(0), \dot{z}_{n}(0)$, excluding the nongeneric initial data identified in Proposition 2.2 for which the system of evolution equations (11) runs into a singularity at a finite time.

These solutions are all nonsingular for all time, remaining in a finite region-the size of which depends on the initial data-of the complex $z$-plane and featuring no particle collisions, i.e., for all time $t$, $z_{n}(t) \neq z_{\ell}(t)$ if $n \neq \ell$; and they are all completely periodic with a period $T$ which is an integer multiple of the basic period $T_{0}$ (see (2b)),

$$
z_{n}(t+T)=z_{n}(t), T=K T_{0},
$$

with the positive integer $K$ restricted as follows: $p \leqslant K \leqslant p N$ !, where, of course, $p$ is the denominator of the rational number $r$ (see (3d)).

Note the arbitrariness of the real (nonvanishing) rational number $r$, and especially of the $N$ complex coupling constants $g_{m}$. In addition, note that Proposition 2.1 implies that all generic solutions of the $\mathrm{N}$-body model characterized by the Newtonian equations of motion (11a)—excluding the nongeneric solutions which are singular (see below Proposition 2.2) - are completely periodic with the same period $T_{M A X}=(p N !) T_{0}$. However, there are lots of solutions that are completely periodic with periods which are integer submultiples of $T_{M A X}$. The detailed identification of these solutions and their periods is a nontrivial matter, as shown, for instance, by the discussion of this phenomenology in the paper [11] — that treats the "periodic goldfish model" (for this terminology, see [8]), which is in 
fact characterized by the same equations of motions (11a), but with all coupling constants vanishing, $g_{m}=0$ - and by the detailed investigation of the structure of the Riemann surfaces associated with other analogous many-body models [21-25].

Proposition 2.2. The solutions of the Newtonian equations of motion (11) may feature singularities via two phenomena, both of which correspond to nongeneric initial data.

The first phenomenon is characterized by initial data satisfying-for at least one value $\bar{m}$ of the index $m$ in its range from 1 to $N$ such that $g_{\bar{m}} \neq 0$ and $-\bar{m} r$ is not a positive integer-the equality

$$
\left|1+\mathbf{i} \omega \check{\tau}_{\bar{m}}^{(1)}\right|=1
$$

where $\check{\tau}_{\bar{m}}^{(1)}$ is defined in terms of the initial data $z_{n}(0), \dot{z}_{n}(0)$ as above (see (12c)).

This singularity occurs at the time $t=\breve{t}^{(1)}$ defined as follows: $\check{t}^{(1)}=\min \left[\check{t}_{\bar{m}}^{(1)}\right]$ (with the minimum taken over the values of the index $\bar{m}$ satisfying the condition (14)), with

$$
\check{t}_{\bar{m}}^{(1)}=\frac{\theta_{\bar{m}}^{(1)}}{\omega}, \check{t}_{\bar{m}}^{(1)}>0,
$$

where $\theta_{\bar{m}}^{(1)}$ is defined $\bmod [2 \pi]$ as follows:

$$
1+\mathbf{i} \omega \check{\tau}_{\bar{m}}^{(1)}=\exp \left(\mathbf{i} \theta_{\bar{m}}^{(1)}\right)
$$

Note that the fact that $\theta_{\bar{m}}^{(1)}$ is real is implied by (14), and that certainly $0<\check{t}_{\bar{m}}^{(1)}<T_{0}$, hence $0<\check{t}^{(1)}<T_{0}$.

The second phenomenon causing the equations of motion (11a) to hit a singularity is the occurrence of a collision of two (or possibly more) particles at some time $t=t_{c}$; so that, for some indices $n, \ell$ with $n \neq \ell$ (of course both in their interval from 1 to $N$ ) there holds the equality $z_{n}\left(t_{c}\right)=z_{\ell}\left(t_{c}\right)$, causing the term $z_{n}(t)-z_{\ell}(t)$ appearing in the denominators in the right-hand side of (11a) to vanish. This phenomenon corresponds to the fact that two of the zeros of the polynomial (4b) with (12) coincide, a fact that clearly only happens for nongeneric initial data (in the complex z-plane), although the condition on the initial data that would cause this phenomenon to happen-and the time at which this phenomenon would happen-can be computed explicitly only for small values of $N$ (and even then the result is not very enlightening).

Let us complete our discussion of the first class of solvable $N$-body problems by displaying the equations of motion (11) in the (simplest) $N=2$ case:

$$
\begin{aligned}
& \ddot{z}_{n}=\mathbf{i}(2 r+1) \omega \dot{z}_{n}+r(r+1) \omega^{2} z_{n}-(-1)^{n}\left(z_{1}-z_{2}\right)^{-1} \\
& \cdot\left\{2\left(\dot{z}_{1}-\mathbf{i} r z_{1}\right)\left(\dot{z}_{2}-\mathbf{i} r z_{2}\right)\right. \\
& +\left(g_{1}\left[\dot{z}_{1}+\dot{z}_{2}-\mathbf{i} r \omega\left(z_{1}+z_{2}\right)\right]^{1+[1 /(1+r)]} z_{n}\right. \\
& \left.\left.-g_{2}\left[\dot{z}_{1} z_{2}+\dot{z}_{2} z_{1}-2 \mathbf{i} r \omega z_{1} z_{2}\right]^{1+[1 /(1+2 r)]}\right)\right\}, n=1,2,
\end{aligned}
$$

with $r$ an arbitrary rational number $(r \neq 0, r \neq-1 / 2, r \neq-1)$, and $g_{1}, g_{2}$ two arbitrary complex numbers (not both vanishing). Note that, for $r=-2 / 3$, both exponents on the right-hand side of these ODEs are integers, since then $1 /(1+r)=3,1 /(1+2 r)=-3$, and this is as well the case for $r=-3 / 4$, since then $1 /(1+r)=4,1 /(1+2 r)=-2$.

Let us end our treatment of the first class of solvable $\mathrm{N}$-body problems characterized by the Newtonian equations of motion (11) by pointing out that, for $r=-2 / 3$ and $N=3$, the exponents on 
the right-hand side of (11a) are all integers, indeed for $r=-2 / 3$, the three exponents $(2+m r) /(1+m r)$ take, for $m=1,2,3$, the three values $4,-2,0$.

The Newtonian equations of motion of the second class of solvable N-body models read as follows:

$$
\begin{aligned}
& \ddot{z}_{n}=\mathbf{i} \omega \dot{z}_{n}+\sum_{\ell=1, \ell \neq n}^{N}\left(\frac{2 \dot{z}_{n} \dot{z}_{\ell}}{z_{n}-z_{\ell}}\right) \\
& -\left[\prod_{\ell=1, \ell \neq n}^{N}\left(z_{n}-z_{\ell}\right)^{-1}\right] \sum_{m=1}^{N}\left[g_{m}\left(\dot{w}_{m}\right)^{2}\left(z_{n}\right)^{N-m}\right],
\end{aligned}
$$

with the various quantities defined as above (in particular $\dot{w}_{m}$ defined in terms of $\dot{z}_{n}$ and $z_{n}$ by (11c) with Notation 1.2). Note that this might be considered the special case of the first class of models (see above) with $r=0, a_{m}=2$ and $b_{m}=0$, which was previously excluded because it requires a special treatment (see Section 3).

The solvable character of this $\mathrm{N}$-body system is demonstrated by the following

Proposition 2.3. The $N$ complex coordinates $z_{n}(t)$ providing the solution at time $t$ of the initial-values problem of the Newtonian equations of motion (17) are the $N$ zeros of the monic polynomial (4b), the $N$ coefficients $w_{m}(t)$ of which are given, in terms of the initial data $z_{n}(0), \dot{z}_{n}(0)$, by the following formula:

$$
w_{m}(t)=w_{m}(0)-\frac{1}{g_{m}} \ln \left[1-\frac{\tau(t)}{\check{\tau}_{m}^{(2)}}\right], \check{\tau}_{m}^{(2)}=\frac{1}{g_{m} \dot{w}_{m}(0)},
$$

of course with $\tau(t)=[\exp (\mathbf{i} \omega t)-1] /(\mathbf{i} \omega)$ (see (2a)). Here and below, the function $\ln \left\{1-\left[\tau(t) / \check{\tau}_{m}^{(2)}\right]\right\}$ is defined by continuity in $t$ from its vanishing value at $t=0$ where $\tau(t)=0$, and, of course, $w_{m}(0)$, respectively, $\dot{w}_{m}(0)$ are defined in terms of the initial data $z_{n}(0)$ and $\dot{z}_{n}(0)$ by (12f), respectively, $(12 \mathrm{~g})$, and if $g_{m}=0$ then (18) becomes $w_{m}(t)=w_{m}(0)+\dot{w}_{m}(0) \tau(t)$, while if $\dot{w}_{m}(0)=0$ it yields $w_{m}(t)=w_{m}(0)$.

Clearly, these coefficients $w_{m}(t)$ are completely periodic in the time $t$ with period $T_{0}$, see (2a),

$$
w_{m}\left(t+T_{0}\right)=w_{m}(t)
$$

iff the initial data satisfy the inequality

$$
\left|1+\mathbf{i} \omega \check{\tau}_{m}^{(2)}\right|>1
$$

iff instead the initial data satisfy the opposite inequality,

$$
\left|1+\mathbf{i} \omega \check{\tau}_{m}^{(2)}\right|<1,
$$

they are periodic in $t$ except for a constant shift (independent of the initial data!) over each period $T_{0}$, so that

$$
w_{m}\left(t+k T_{0}\right)=w_{m}(t)-\frac{2 \pi \mathbf{i} k}{g_{m}}, k= \pm 1, \pm 2, \pm 3, \ldots
$$

hence, in this second case, they diverge as $t \rightarrow \pm \infty$. In addition-in the intermediate, nongeneric case in which the initial data imply the equality

$$
\left|1+\mathbf{i} \omega \check{\tau}_{m}^{(2)}\right|=1
$$


- the coefficient $w_{m}(t)$ diverges at the finite times

$$
\check{t}_{m}^{(2)}=(\mathbf{i} \omega)^{-1} \ln \left(1+\mathbf{i} \omega \check{\tau}_{m}^{(2)}\right) \bmod \left(T_{0}\right),
$$

which are, of course, real thanks to (21a).

Correspondingly, the particles coordinates $z_{n}(t)$-being the $N$ zeros of the polynomial $(4 \mathrm{~b})$ with the coefficients $w_{m}(t)$ (see (18))-are periodic with period $K T_{0}-K$ being a positive integer in the range from 1 to $N$ ! (see below Remark 3.1) -iff the initial data satisfy the inequality (19b), while iff instead the initial data satisfy the opposite inequality (20a) at least one of the particle coordinates $z_{n}(t)$ comes from or escapes to infinity in the remote past and future: see, for instance, the relevant discussion in Appendix $G$ ("Asymptotic behavior of the zeros of a polynomial whose coefficients diverge exponentially") of the book [9]. In addition, of course, if the (nongeneric) initial data imply validity of the equality (21a), the equations of motion run into a singularity at $t=\breve{t}_{m}^{(2)}$ (see (21b)). Other nongeneric initial data causing the equations of motion (17) to run into a singularity at a finite time are those leading to particle collisions. Note that generally the nongeneric initial data causing singularities are also those that separate the regions of initial data associated to different behaviors of the model, including the emergence of the higher periodicities associated to values of $K$ larger than unity as well as the periodic and nonperiodic cases.

We do not display explicitly the equations of motion of this second model in the simplest $N=2$ case because they can be immediately obtained by setting $r=0$ in those of the first model (see (16)).

We conclude our report on the properties of the second class of solvable $N$-body problems with the following remark (proven in Section 3):

Remark 2.1. The system of Newtonian equations of motion (17) is Hamiltonian, albeit with a time-dependent Hamiltonian.

The third class of solvable $\mathrm{N}$-body problems is characterized by the Newtonian equations of motion

$$
\begin{aligned}
& \ddot{z}_{n}=\mathbf{i}(2 r+1) \omega \dot{z}_{n}+r(r+1) \omega^{2} z_{n} \\
& +\sum_{\ell=1, \ell \neq n}^{N}\left[\frac{2\left(\dot{z}_{n}-\mathbf{i} r \omega z_{n}\right)\left(\dot{z}_{\ell}-\mathbf{i} r \omega z_{\ell}\right)}{z_{n}-z_{\ell}}\right]-\left[\prod_{\ell=1, \ell \neq n}^{N}\left(z_{n}-z_{\ell}\right)^{-1}\right] \\
& \cdot \sum_{m=1}^{N}\left[g_{m}\left(\dot{w}_{m}-\mathbf{i} m r w_{m}\right)^{2-\frac{1}{1+m r}}\left(w_{m}\right)^{-1+\frac{1}{m r}}\left(z_{n}\right)^{N-m}\right],
\end{aligned}
$$

where $\omega$ is an arbitrary nonvanishing real number, $r$ is an arbitrary rational number (of course if $g_{m} \neq 0$ then $r \neq 0$ and $r \neq 1 / m$ for the integer $m$ in its range from 1 to $N$ ), the $N$ coupling constants $g_{m}$ are arbitrary complex numbers (not all vanishing), and the quantities $w_{m} \equiv w_{m}(t)$, respectively, $\dot{w}_{m} \equiv \dot{w}_{m}(t)$ are expressed in terms of the coordinates $z_{n} \equiv z_{n}(t)$ and $\dot{z}_{n} \equiv \dot{z}_{n}(t)$ via (11b), respectively (11c).

The solvable character of this $N$-body problem is demonstrated by the following

Proposition 2.4. The $N$ complex coordinates $z_{n}(t)$ providing the solution at time $t$ of the initial-values problem of the Newtonian equations of motion (22) are the $N$ zeros of the monic polynomial (4b), the $N$ coefficients $w_{m}(t)$ of which are given, in terms of the initial data $z_{n}(0), \dot{z}_{n}(0)$, by the following formulas:

$$
\begin{gathered}
w_{m}(t)=\exp (\mathbf{i} m r \omega t) \gamma_{m}(\tau(t)), \tau(t)=\frac{\exp (\mathbf{i} \omega t)-1}{\mathbf{i} \omega}, \\
\gamma_{m}(\tau)=\left[B_{m}^{(3)}\right]^{m r}\left\{\left[\frac{\check{\tau}_{m}^{(3)}-\hat{\tau}_{m}^{(3)}}{\check{\tau}_{m}^{(3)}-\tau}\right]^{\frac{1}{m r}}-1\right\}^{-m r},
\end{gathered}
$$




$$
\begin{gathered}
B_{m}^{(3)}=\frac{\left[\dot{w}_{m}(0)-\mathbf{i} m r \omega w(0)\right]^{\frac{1}{1+m r}}}{A_{m}}-[w(0)]^{\frac{1}{m r}}, A_{m}=\frac{g_{m} m r}{1+m r}, \\
\check{\tau}_{m}^{(3)}=-\frac{\left\{1+B_{m}^{(3)}[w(0)]^{-\frac{1}{m r}}\right\}^{-m r}}{\left(A_{m}\right)^{1+m r} B_{m}^{(3)}}, \\
\hat{\tau}_{m}^{(3)}=\check{\tau}_{m}^{(3)}\left\{1-\left(1+B_{m}^{(3)}[w(0)]^{-\frac{1}{m r}}\right)^{m r}\right\},
\end{gathered}
$$

where $w_{m}(0)$, respectively, $\dot{w}_{m}(0)$, are defined in terms of the initial data $z_{n}(0)$ and $\dot{z}_{n}(0)$ by (12f), respectively, $(12 \mathrm{~g})$, and of course the determinations of the rational roots are implied by the requirement that these formulas be valid at the initial time $t=0$ and thereafter by continuity in $t$, and again if $g_{m}=0$, the expression (23b) must be replaced by (12h).

It is plain that, if neither $m r$ nor $1 /(m r)$ are integers, for generic initial data the function $\gamma_{m}(\tau)$ features, in the complex $\tau$-plane, two rational branch points at $\tau=\check{\tau}_{m}^{(3)}$, respectively, at $\tau=\hat{\tau}_{m}^{(3)}$, and that the periodicity of $\gamma_{m}(t) \equiv \gamma_{m}(\tau(t))$ as function of $t$ (see (23b)), is determined by the location in the complex $\tau$-plane of these two branch points with respect to the circle $\tilde{C}$ centered at $\tau=\mathbf{i} / \omega$ and with radius $1 /|\omega|$ on which rotates the point $\tau(t)=[\exp (\mathbf{i} \omega t)-1] /(\mathbf{i} \omega)$ as function of the time $t$. If both branch points are located outside the circle $\tilde{C}$ - and the condition on the initial data determining this outcome is clearly validity of both inequalities

$$
\left|1+\mathbf{i} \omega \check{\tau}_{m}^{(3)}\right|>1,\left|1+\mathbf{i} \omega \hat{\tau}_{m}^{(3)}\right|>1,
$$

with $\check{\tau}_{m}^{(3)}$, respectively, $\hat{\tau}_{m}^{(3)}$ defined in terms of the initial data by (23d), respectively, (23e)—then clearly $\gamma_{m}(t) \equiv \gamma_{m}(\tau(t))$ as a function of $t$ is periodic with period $T_{0}$, see (2a),

$$
\gamma_{m}\left(t+T_{0}\right)=\gamma_{m}(t),
$$

hence $w_{m}(t)$ is periodic in $t$ with period $T_{\hat{p}_{m}}=\hat{p}_{m} T_{0}$,

$$
w_{m}\left(t+T_{\hat{p}_{m}}\right)=w_{m}(t),
$$

with $\hat{p}_{m}=p / m$ if this number is integer; otherwise, $\hat{p}_{m}=p$ (where of course $p$ is the denominator of $r$ : see (23a) and (3d)). In addition, as a consequence-if the inequalities (24a) are valid for all values of $m$ in the range from 1 to $N$-then the polynomial $(4 \mathrm{~b})$ is periodic in $t$ with period

$$
T=T_{0}\left\{\underset{\bar{m}=1,2, \ldots, N}{\left.\operatorname{MinimumCommonMultiple}\left[\hat{p}_{\bar{m}}\right]\right\}},\right.
$$

where the MinimumCommonMultiple must be evaluated for the values of $m=\bar{m}$ such that $g_{\bar{m}} \neq 0$; hence (see below Remark 3.1), the $N$ coordinates $z_{n}(t)$ are periodic with period $Q T$, where $Q$ is a positive integer in the range from 1 to $N$ !.

It is also plain that the solutions $z_{n}(t)$ are periodic in $t$ with a period which is a positive integer multiple $\tilde{Q}$ of $T_{0}$ even if the initial data imply instead that some of the inequalities (24a) are reversed, but, in these cases, the determination of the outcome-in particular, of the value of $\tilde{Q}$-requires, to begin with, a standard analysis of the structure of the Riemann surface associated to the function

$$
\varphi_{m}(\tau)=\left\{\left[\frac{\check{\tau}_{m}^{(3)}-\hat{\tau}_{m}^{(3)}}{\check{\tau}_{m}^{(3)}-\tau}\right]^{\frac{1}{m r}}-1\right\}^{-m r}
$$

of the complex variable $\tau$, and consequently of the periodicity of $\varphi_{m}(t) \equiv \varphi_{m}(\tau(t))$ as a function of the real variable $t$ ("time") when $\tau(t)=[\exp (\mathbf{i} \omega t)-1] /(\mathbf{i} \omega)$ so that $\tau(t)$ travels on the circle $\tilde{C}$ with 
radius $1 /|\omega|$ centered at $\tau=\mathbf{i} / \omega$; and then an analysis of the resulting periodicity of the solutions $z_{n}(t)$ analogous to that made in Section 3 for the first class of solvable models. We leave this task to the interested reader.

Let us complete this discussion of the third class of solvable $N$-body problems by displaying the equations of motion (22) in the (simplest) $N=2$ case:

$$
\begin{aligned}
& \ddot{z}_{n}=\mathbf{i}(2 r+1) \omega \dot{z}_{n}+r(r+1) \omega^{2} z_{n}-(-1)^{n}\left[z_{1}-z_{2}\right]^{-1} \\
& \cdot\left\{2\left(\dot{z}_{1}-\mathbf{i} r z_{1}\right)\left(\dot{z}_{2}-\mathbf{i} r z_{2}\right)\right. \\
& -g_{1}\left[-\dot{z}_{1}-\dot{z}_{2}+\mathbf{i} r\left(z_{1}+z_{2}\right)\right]^{2-\frac{1}{1+r}}\left[-\left(z_{1}+z_{2}\right)\right]^{-1+\frac{1}{r}} z_{n} \\
& \left.-g_{2}\left(\dot{z}_{1} z_{2}+z_{1} \dot{z}_{2}-2 \mathbf{i} r z_{1} z_{2}\right)^{2-\frac{1}{1+2 r}}\left(z_{1} z_{2}\right)^{-1+\frac{1}{2 r}}\right\}, n=1,2
\end{aligned}
$$

with $r$ an arbitrary rational number $(r \neq 0, r \neq-1 / 2, r \neq-1)$, and $g_{1}, g_{2}$ two arbitrary complex numbers (not both vanishing).

In addition, let us end this discussion of the third class of solvable $N$-body problems characterized by the Newtonian equations of motion (22) by also displaying these equations in the following two special cases:

case (i), with $r=-1 / 2$ and $g_{m}=0$ for $m>1$, when these equations read

$$
\begin{aligned}
& \ddot{z}_{n}=-\frac{1}{4} \omega^{2} z_{n}+\sum_{\ell=1, \ell \neq n}^{N}\left[\frac{\left(2 \dot{z}_{n}+\mathbf{i} \omega z_{n}\right)\left(2 \dot{z}_{\ell}+\mathbf{i} \omega z_{\ell}\right)}{2\left(z_{n}-z_{\ell}\right)}\right] \\
& +g_{1}\left(z_{n}\right)^{N-1}\left[\prod_{\ell=1, \ell \neq n}^{N}\left(z_{n}-z_{\ell}\right)^{-1}\right]\left[\sum_{n_{1}=1}^{N}\left(z_{n_{1}}\right)\right]^{-3} ;
\end{aligned}
$$

case (ii), with $r=-1 / 4$ and $g_{m}=0$ for $m \neq 2$, when these equations of motion read

$$
\begin{aligned}
& \ddot{z}_{n}=\frac{1}{2} \mathbf{i} \omega \dot{z}_{n}-\frac{3}{16} \omega^{2} z_{n}+\sum_{\ell=1, \ell \neq n}^{N}\left[\frac{\left(4 \dot{z}_{n}+\mathbf{i} \omega z_{n}\right)\left(4 \dot{z}_{\ell}+\mathbf{i} \omega z_{\ell}\right)}{8\left(z_{n}-z_{\ell}\right)}\right] \\
& -\frac{1}{2} g_{2}\left(z_{n}\right)^{N-2}\left[\prod_{\ell=1, \ell \neq n}^{N}\left(z_{n}-z_{\ell}\right)^{-1}\right]\left[\sum_{n_{1}, n_{2}=1 ; n_{1} \neq n_{2}}^{N}\left(z_{n_{1}} z_{n_{2}}\right)\right]^{-3} .
\end{aligned}
$$

\section{Proofs}

In this Section 3 we prove the results reported without their proofs in the preceding Section 2.

The first task is to integrate once the ODE (10a). This is an easy task, yielding

$$
\begin{gathered}
\gamma_{m}^{\prime}(\tau)=\left\{A_{m}\left(\left[\gamma_{m}(\tau)\right]^{1+b_{m}}+B_{m}\right)\right\}^{\frac{1}{2-a_{m}}}, \\
A_{m}=\frac{g_{m}\left(2-a_{m}\right)}{1+b_{m}}=\frac{g_{m} m r}{1+m r}, \\
B_{m}=\frac{\left[\gamma_{m}^{\prime}(0)\right]^{2-a_{m}}}{A_{m}}-\left[\gamma_{m}(0)\right]^{1+b_{m}} .
\end{gathered}
$$

Note that, above and hereafter, we exclude from consideration the special case with $a_{m}=2$ and $b_{m}=-1$, which is treated in [6]. 
The next integration can be performed in terms of elementary functions only for special assignments (satisfying the restriction (10b)) of the parameters $a_{m}$ and $b_{m}$, to which we restrict attention in the present paper.

Our first assignment is

$$
b_{m}=0
$$

implying via (10b)

$$
a_{m}=2-\frac{m r}{1+m r}=\frac{2+m r}{1+m r}
$$

and

$$
\gamma_{m}^{\prime}(\tau)=\left\{A_{m}\left[\gamma_{m}(\tau)+B_{m}^{(1)}\right]\right\}^{1+\frac{1}{m r}}
$$

with $A_{m}$, respectively, $B_{m}^{(1)}$ defined by (28b) (or, equivalently, (12d)), respectively, (12e). Here, we assume, of course, that $r \neq 0$ (implying $a_{m} \neq 2$ ) and moreover that $r \neq-1 / m$ with $m=1,2, \ldots, N$ (implying that $a_{m}$ is a finite rational number for all values of $m$ in its range from 1 to $N$ ).

This ODE (29c) can now be easily integrated, yielding (12b) with (12c).

These developments clearly prove the first part of Proposition 2.1.

To prove the second part of Proposition 2.1 we ascertain, to begin with, the periodicity properties as functions of the time variable $t$ of the coefficients $\gamma_{m}(t) \equiv \gamma_{m}(\tau(t))$ (see (12b)). The starting point is the observation that $\tau(t)$-see (2) or (12a)—is a periodic function of $t$ with period $T_{0}$, rotating in the complex $\tau$-plane on the circle $\tilde{C}$ centered at the point $\mathbf{i} / \omega$ and having radius $1 /|\omega|$. Hence, any holomorphic function of $\tau$ is as well periodic in $t$ with period $T_{0}$; this clearly is (for all values of $m$ in its range from 1 to $N$ ) the case of the functions $\gamma_{m}(\tau)$ ( see (12b)), if $r$ is a negative integer. If instead $r$ is a positive integer, the functions $\gamma_{m}(\tau)$ are meromorphic in $\tau$, featuring a polar singularity at $\tau=\check{\tau}_{m}^{(1)}$ ( see (12b)). In this case, $\gamma_{m}(t) \equiv \gamma_{m}(\tau(t))$ is again generally periodic in $t$ with period $T_{0}$, $\gamma_{m}\left(t+T_{0}\right)=\gamma_{m}(t)$, but for the nongeneric assignments of the initial data such that $\check{\tau}_{m}^{(1)}$ falls on the circle $\tilde{C}$-note that $\check{\tau}_{m}^{(1)}$ does depend on the initial data (see (12c) and (12e)), and that the condition for this to happen is validity of the equality

$$
\left|1+\mathbf{i} \omega \check{\tau}_{m}^{(1)}\right|=1
$$

then the function $\gamma_{m}(t) \equiv \gamma_{m}(\tau(t))$ diverges at the times

$$
\check{t}_{m}^{(1)}=(\mathbf{i} \omega)^{-1} \ln \left(1+\mathbf{i} \omega \check{\tau}_{m}^{(1)}\right) \bmod \left(T_{0}\right) .
$$

Finally, if $r$ is rational but not integer, i.e., $p>1$ (see (3d)), and $m$ is not an integer multiple of $p$, $\gamma_{m}(\tau)$ features a rational branch point at $\tau=\check{\tau}_{m}^{(1)}$ (see (12b)), then the evolution of $\gamma_{m}(t) \equiv \gamma_{m}(\tau(t))$ as function of the time $t$ depends on the location of the branch point $\check{\tau}_{m}^{(1)}$ in the complex $\tau$-plane, whether it falls outside, inside or just on the circle $\tilde{C}$. The latter case requires that the initial data satisfy the condition (30a), implying again that they are not generic. While clearly the condition that the branch point be located outside the circle $\tilde{C}$ is validity of the inequality

$$
\left|1+\mathbf{i} \omega \check{\tau}_{m}^{(1)}\right|>1,
$$

and for the corresponding nongeneric initial data the function $\gamma_{m}(t) \equiv \gamma_{m}(\tau(t))$ is again periodic with period $T_{0}$. If instead the branch point falls inside the circle $\tilde{C}$, and the condition on the initial data for this to happen is validity of the opposite inequality

$$
\left|1+\mathbf{i} \omega \check{\tau}_{m}^{(1)}\right|<1
$$


the periodicity of $\gamma_{m}(t) \equiv \gamma_{m}(\tau(t))$ gets modified: the period is then $\tilde{T}_{p}=p T_{0}$ (see (12b) and (3d)), unless $p / m=\breve{p}_{m}$ is an integer, in which case the period is $\check{T}_{\check{p}_{m}}=\breve{p}_{m} T_{0}$.

Next, let us discuss the $t$-periodicity of $w(t)$, related to $\gamma(t) \equiv \gamma(\tau(t))$ by (8a) or (12a). We then note that the prefactor $\exp (\mathbf{i} r m \omega t)$ in (8a) or (12a) is also periodic in $t$ (see (3d)), with the same period $\check{T}_{\check{p}_{m}}=\check{p}_{m} T_{0}$, where $\check{p}_{m}=p$ unless $p / m$ is an integer, in which case $\check{p}_{m}=p / m$. We may therefore conclude that, for all generic initial data, the functions $w_{m}(t)$ are periodic with period $\check{T}_{\check{p}_{m}}$,

$$
w_{m}\left(t+\check{T}_{\check{p}_{m}}\right)=w_{m}(t) .
$$

Next, let us discuss the periodicity of the zeros $z_{n}(t)$ of the polynomial $(4 \mathrm{~b})$ with coefficients $w_{m}(t)$ periodic as indicated just above. In this context, the following Remark 3.1 is relevant.

Remark 3.1. If a time-dependent polynomial $P_{N}(z ; t)$, of degree $N$ in $z$, is time-periodic with period $\tilde{T}$, $P_{N}(z ; t+\tilde{T})=P_{N}(z ; t)$, the unordered set $\underline{z}(t)$ of its $N$ zeros $z_{n}(t)$ is of course periodic with the same period $\tilde{T}, \underline{z}(t+\tilde{T})=\underline{z}(t)$ (since after a period the polynomial is unchanged); however, due to the possibility that these zeros, as it were, "exchange their places" over their time evolution, the period of each individual zero $z_{n}(t)$, considered as a continuous function of time, may be a positive integer multiple of $\tilde{T}$; although of course that multiple cannot exceed the number $N$ ! of permutations of the $N$ elements of the unordered set $\underline{z}(t)$ (for a detailed discussion of this phenomenology in analogous many-body contexts see [11,21-25]).

We can therefore conclude that the $N$ zeros $z_{n}(t)$ of the monic polynomial $(4 \mathrm{~b})$, the $N$ coefficients $w_{m}(t)$ of which are periodic as described above, are also periodic, for generic initial data, with periods $K T_{0}$, with the positive integer $K$ restricted as follows:

$$
p \leqslant K \leqslant N ! p
$$

since the very definition of $\breve{p}_{m}$ (see above) implies that $p$ is the Minimum Common Multiple of the $N$ parameters $\check{p}_{m}$.

This ends the proofs of the findings reported in Propositions 2.1 and 2.2.

The proofs of the other two Propositions reported in Section 2 follow, but below we omit the proofs of some aspects of the results reported in Section 2, which are analogous to those detailed above in the formulations and proofs of Propositions 2.1 and 2.2.

Hence, the only aspect relevant to prove Proposition 2.3 that we do report is the derivation of the formula (18). The starting point is the ODE

$$
\gamma_{m}^{\prime \prime}=g_{m}\left(\gamma_{m}^{\prime}\right)^{2}
$$

which is the special case of (10a) with $r=0, a_{m}=2$ and $b_{m}=0$. The integration of this ODE is a trivial task, yielding

$$
\gamma_{m}(\tau)=\gamma_{m}(0)-\frac{\ln \left[1-g_{m} \gamma_{m}^{\prime}(0) \tau\right]}{g_{m}},
$$

and the fact that this implies (18) is an immediate consequence of (8) with $r=0$.

As for the proof of Remark 2.1, it is based on the observation that the decoupled nonlinear system of $N$ ODEs

$$
\ddot{w}_{m}=i \omega \dot{w}_{m}+g\left(\dot{w}_{m}\right)^{2}
$$

is Hamiltonian, since it is implied by the standard Hamiltonian equations of motion

$$
\dot{w}=\frac{\partial H(\vec{v}, \vec{w} ; t)}{\partial v_{m}}, \dot{v}=-\frac{\partial H(\vec{v}, \vec{w} ;)}{\partial w_{m}},
$$


with the (time-dependent) Hamiltonian

$$
H(\vec{v}, \vec{w} ; t)=\exp (i \omega t) v^{\alpha} \exp (g \alpha w),
$$

as the diligent reader will easily verify. Note the arbitrariness of the (nonvanishing!) parameter $\alpha$.

However, via the two identities

$$
\begin{gathered}
\dot{z}_{n}=-\left[\prod_{\ell=1, \ell \neq n}^{N}\left(z_{n}-z_{\ell}\right)^{-1}\right] \sum_{m=1}^{N}\left[\dot{w}\left(z_{n}\right)^{N-m}\right], \\
\ddot{z}_{n}=\sum_{\ell=1, \ell \neq n}^{N}\left(\frac{2 \dot{z}_{n} \dot{z}_{\ell}}{z_{n}-z_{\ell}}\right)-\left[\prod_{\ell=1, \ell \neq n}^{N}\left(z_{n}-z_{\ell}\right)^{-1}\right] \sum_{m=1}^{N}\left[\ddot{w}\left(z_{n}\right)^{N-m}\right],
\end{gathered}
$$

linking —as proven in [1] — the time evolutions of the $N$ zeros $z_{n}(t)$ and of the $N$ coefficients $w_{m}(t)$ of the monic time-dependent polynomial (4b), it is easily seen that the set of ODEs (36a) correspond precisely to the equations of motion (17). This implies that the equations of motion (17) are also Hamiltonian, since the $N$ coordinates $z_{n}$ are linked to the $N$ canonical coordinates $w_{m}$ by the "point" transformations-not involving the canonical momenta $v_{m}$ - which relate the coefficients and the zeros of a polynomial (see (7) or (11b)).

Let us finally deal with the third class of $N$-body models, obtained by identifying another set of parameters $r, a_{m}, b_{m}$ allowing the ODEs (28) to be explicitly integrated in terms of elementary functions. To this end, we introduce the auxiliary functions

$$
\eta_{m}(\tau)=\left[\gamma_{m}(\tau)\right]^{s_{m}}, \gamma_{m}(\tau)=\left[\eta_{m}(\tau)\right]^{1 / s_{m}}
$$

with the option to assign the parameters $s_{m}$ at our convenience (see below). We then see, from (28) with (10b), that these auxiliary functions satisfy the ODEs

$$
\begin{gathered}
\eta_{m}^{\prime}=s_{m}\left[A_{m}\left(\eta^{\alpha_{m}}+B_{m} \eta^{\beta_{m}}\right)\right]^{\frac{1}{2-a_{m}}}, \\
\alpha_{m}=\frac{b_{m}+1+\left(s_{m}-1\right)\left(2-a_{m}\right)}{s_{m}}, \beta_{m}=\frac{\left(s_{m}-1\right)\left(2-a_{m}\right)}{s_{m}},
\end{gathered}
$$

with $A_{m}$, respectively, $B_{m}$ defined by (28b), respectively, (28c). Thus, we set $\alpha_{m}=0$ and $\beta_{m}=1$, implying (after a bit of elementary algebra)

$$
s_{m}=-\frac{1}{m r}, b_{m} \equiv b_{m}^{(3)}=-1+\frac{1}{m r}, \quad a_{m} \equiv a_{m}^{(3)}=2-\frac{1}{1+m r} .
$$

The ODE (38b) then reads

$$
\eta_{m}^{\prime}=-\frac{1}{m r}\left[A_{m}\left(1+B_{m}^{(3)} \eta_{m}\right)\right]^{1+m r}
$$

with $A_{m}$, respectively, $B_{m}^{(3)}$ defined by (28b) respectively (28c) with (39), while the assignments of the parameters $r$ and $g_{m}$ are still free (of course $r \neq 0, r \neq-1 / m$ ). This ODE, (40), can then be immediately integrated, yielding

$$
\begin{gathered}
\eta_{m}(\tau)=\left[\frac{1}{B_{m}^{(3)}}+\eta_{m}(0)\right]\left(1-\frac{\tau}{\check{\tau}_{m}^{(3)}}\right)^{-\frac{1}{m r}}-\frac{1}{B_{m}^{(3)}} \\
B_{m}^{(3)}=\frac{\left[\gamma^{\prime}(0)\right]^{\frac{1}{1+m r}}}{A_{m}}-[\gamma(0)]^{\frac{1}{m r}}
\end{gathered}
$$




$$
\check{\tau}_{m}^{(3)}=-\frac{\left[1+B_{m}^{(3)} \eta_{m}(0)\right]^{-m r}}{\left(A_{m}\right)^{1+m r} B_{m}^{(3)}} .
$$

Hence, finally, via (38a) with (39) and (8), we arrive at the formulas (23), thereby proving Proposition 2.4 .

\section{Outlook}

In this Section 4 we outline tersely possible extensions of the results obtained in this paper. A tentative list of such possible developments is provided in the last Section of [6]: the first of those reported there is essentially implemented in the present paper, while the others are still open. Also open is the possibility to investigate a combination of the three classes of models treated above, and of the class of models treated in [6], by assuming that, for different values of the parameter $m$, some coefficients $\gamma_{m}$ evolve according to the three different solvable subcases of the ODEs (28) discussed above in Section 3 or to the case discussed in [6]. In addition, of course, also open is the investigation of the hierarchies of solvable models associated with each of those mentioned herein via the notion of generations of monic polynomials such that the coefficients of the polynomials of the next generation coincide with the zeros of a polynomial of the current generation [2].

Other $\mathrm{N}$-body problems-more general than those treated in [6] and in the present paper-can be investigated by analogous techniques to those employed above, while allowing each $\gamma_{m}(t)$ to satisfy a solvable second-order ODE still belonging to the class (10) but forsaking the restriction on the parameters $a_{m}$ and $b_{m}$ necessary and sufficient to allow the solution of these ODEs in terms of elementary functions (for instance, a natural extension might include special functions of elliptic and hyperelliptic type).

Conflicts of Interest: The author declares no conflict of interest.

\section{References}

1. Calogero, F. New solvable variants of the goldfish many-body problem. Studies Appl. Math. 2016, doi:10.1111/sapm.12096.

2. Bihun, O.; Calogero, F. Generations of monic polynomials such that the coefficients of each polynomial of the next generation coincide with the zeros of a polynomial of the current generation, and new solvable many-body problems. Lett. Math. Phys. 2016, 106, 1011-1031.

3. Bihun, O.; Calogero, F. A new solvable many-body problem of goldfish type. J. Nonlinear Math. Phys. 2016, 23, 28-46.

4. Bihun, O.; Calogero, F. Novel solvable many-body problems. J. Nonlinear Math. Phys. 2016, 23, $190-212$.

5. Calogero, F. A solvable $N$-body problem of goldfish type featuring $N^{2}$ arbitrary coupling constants. J. Nonlinear Math. Phys. 2016, 23, 300-305.

6. Calogero, F. Novel isochronous $N$-body problems featuring $N$ arbitrary rational coupling constants. J. Math. Phys. 2016, in press.

7. Calogero, F. Motion of poles and zeros of special solutions of nonlinear and linear partial differential equations, and related "solvable" many-body problems. II Nuovo Cimento B 1978, 43, 177-241.

8. Calogero, F. The neatest many-body problem amenable to exact treatments (a "goldfish"?). Phys. D Nonlinear Phenom. 2001, 152-153, 78-84.

9. Calogero, F. Classical many-body problems amenable to exact treatments; Springer: Heidelberg, Germany, 2001; p. 749.

10. Nucci, M.C. Calogero's 'goldfish' is indeed a school of free particles. J. Phys. A Math. Gen. 2004, 37, 11391-11400.

11. Gómez-Ullate, D.; Sommacal, M. Periods of the goldfish many-body problem. J. Nonlinear Math. Phys. 2005, 12, 351-362.

12. Suris, Y.B. Time discretization of F. Calogero's "Goldfish" System. J. Nonlinear Math. Phys. 2005, $12,633-647$. 
13. Calogero, F.; Iona, S. Novel solvable extensions of the goldfish many-body model. J. Math. Phys. 2005, 46, 103515.

14. Guillot, A. The Painlevé property for quasihomogeneous systems and a many-body problem in the plane. Comm. Math. Phys. 2005, 256, 181-194.

15. Bruschi, M.; Calogero, F. Novel solvable variants of the goldfish many-body model. J. Math. Phys. 2006, 47, 022703.

16. Calogero, F.; Langmann, E. Goldfishing by gauge theory. J. Math. Phys. 2006, 47, 082702.

17. Arlind, J.; Bordemann, M.; Hoppe, J.; Lee, C. Goldfish geodesics and Hamiltonian reduction of matrix dynamics. Lett. Math. Phys. 2008, 84, 89-98.

18. Calogero, F. Isochronous Systems; Oxford University Press: Oxford, UK, 2008; p. 264.

19. Bihun, O.; Calogero, F. Solvable many-body models of goldfish type with one-, two- and three-body forces. SIGMA 2013, 9, 059.

20. Jairuk, U.; Yoo-Kong, S.M.; Tanasittikosol, M. The Lagrangian structure of Calogero's goldfish model. Theor. Math. Phys. 2015, 183, 665-683.

21. Calogero, F.; Gómez-Ullate, D.; Santini, P.M.; Sommacal, M. The transition from regular to irregular motions, explained as travel on Riemann surfaces. J. Phys. A Math. Gen. 2005, 38, 8873-8896.

22. Sommacal, M. The Transition from Regular to Irregular Motion, Explained as Travel on Riemann Surfaces. Ph.D. Thesis, SISSA, Trieste, Italy, 2005.

23. Grinevich, P.G.; Santini, P.M. Newtonian dynamics in the plane corresponding to straight and cyclic motions on the hyperelliptic curve: Ergodicity, isochrony and fractals. Phys. D Nonlinear Phenom. 2007, 232, $22-32$.

24. Calogero, F.; Gómez-Ullate, D.; Santini, P.M.; Sommacal, M. Towards a theory of chaos explained as travel on Riemann surfaces. J. Phys. A. Math. Theor. 2009, 42, 015205.

25. Gómez-Ullate, D.; Santini, P.M.; Sommacal, M.; Calogero, F. Understanding complex dynamics by means of an associated Riemann surface. Phys. D Nonlinear Phenom. 2012, 241, 1291-1305.

(C) 2016 by the author; licensee MDPI, Basel, Switzerland. This article is an open access article distributed under the terms and conditions of the Creative Commons Attribution (CC-BY) license (http://creativecommons.org/licenses/by/4.0/). 